\title{
Prolonged Seed Dormancy in Phyllanthus emblica L. Can Be Overturned by Seed Scarification and Gibberellin Pre Treatment
}

\author{
S. M. U. P. Mawalagedera ${ }^{1}$, G. A. D. Perera ${ }^{2}$, S. D. S. S. Sooriyapathirana ${ }^{1}$ \\ ${ }^{1}$ Department of Molecular Biology and Biotechnology, Faculty of Science, \\ University of Peradeniya, Peradeniya, Sri Lanka \\ ${ }^{2}$ Department of Botany, Faculty of Science, University of Peradeniya, Peradeniya, Sri Lanka \\ Email: sunethss09@gmail.com
}

Received September $26^{\text {th }}, 2013$; revised November $3^{\text {rd }}, 2013$; accepted November $26^{\text {th }}, 2013$

\begin{abstract}
Copyright () 2014 S. M. U. P. Mawalagedera et al. This is an open access article distributed under the Creative Commons Attribution License, which permits unrestricted use, distribution, and reproduction in any medium, provided the original work is properly cited. In accordance of the Creative Commons Attribution License all Copyrights (C) 2014 are reserved for SCIRP and the owner of the intellectual property S. M. U. P. Mawalagedera et al. All Copyright $(\underset{0}{2014}$ are guarded by law and by SCIRP as a guardian.
\end{abstract}

Phyllanthus emblica L. is an important constituent of Ayurvedic medicine and a fresh fruit species in the market in Sri Lanka. Therefore, it has a high potential to be established as a commercial fruit crop. The seeds of $P$. emblica are semi orthodox and exhibit a long dormancy period hindering the natural sexual propagation. Therefore, it still remains as an underutilized fruit crop in Sri Lanka due to its lack of quality planting material and poor propagation techniques. Long term dormancy also causes a big challenge in germinating seeds to create populations in breeding programs. In order to promote $P$. emblica from its underutilized status, what seems most feasible is to develop a method to break up the seed dormancy artificially. In order to do so, the methods of breaking the dormancy of $P$. emblica seeds have to be studied. Therefore, the aim of this study was to identify a method to break up the seed dormancy of $P$. emblica. The seeds were extracted from 21 trees belonging to three different districts in Sri Lanka. The selected viable seeds were subjected to four different pre treatments: none treated seeds (i.e. control), seeds scarified, seeds scarified and treated with $1 \%$ gibberellin and seed coat removed and followed by seeds treated with $1 \%$ gibberellin. From the four treatments, seed dormancy was overturned with a germination percentage of $43 \%$ by the seed pre treatment where the seeds were scarified and treated with $1 \%$ gibberellin and no other pre treatment methods were successful in breaking the dormancy. This suggests that the natural germination potential of $P$. emblica seeds is very low and it can be overridden by seed scarification and gibberellin pre treatment.

Keywords: Gibberellin Pre Treatment; Phyllanthus emblica; Seed Dormancy; Seed Scarification

\section{Introduction}

Phylanthus emblica L. is a deciduous and monoecious tree belonging to family Euphorbiaceae. Fruit is fleshy and drupaceous and the seeds are found within the hardened endocarp of the fruit known as stone (Dassanayake \& Fosberg, 1988). $P$. emblica is famous for its medicinal values. In traditional medicine, it is known as one of "the best rejuvenating herbs" (Krishnaveni \& Mirunalini, 2011). All the parts of the tree are medically important (Pushpakumara et al., 2007), but the drupe is the most important. There is a significant market demand for fresh drupes because it relieves fatigue, increases the appetite and also acts as a good purgative (Krishnaveni \& Mirunalini, 2011). The pharmacological studies indicated that drupes show antioxidant and antiproliferative activities attributed to the phenolic compounds in the drupes (Lou et al., 2011), highlighting the importance of $P$. emblica. However, it is still an underutilized fruit crop in Sri Lanka due to unavailability of high quality planting material and poor propagation techniques (Pushpakumara et al., 2007).

The seeds of $P$. emblica exhibit a long dormancy period. The observations indicated that the natural sexual propagation po- tential of $P$. emblica is very poor and the seed behaviors as semi orthodox (Pushpakumara et al., 2007). Under natural conditions, $P$. emblica seeds are dispersed either through ruminants (Prasad et al., 2006) or by passive means. But there is a high tendency towards passive means of dispersal. Therefore, under natural conditions, this semi orthodox behavior and long-term dormancy aid the seeds to remain viable until the seeds come into contact with the proper substratum for germination. But in terms of agronomy, this is a major drawback in implementing large scale P. emblica plantations (Pushpakumara et al., 2007). Also in setting up breeding programs, long-term dormancy causes a big challenge in germinating seeds to create populations. Thus, there is a need to develop a method to break up the seed dormancy artificially. In order to do so, the methods available to break the dormancy of seeds have to be studied extensively for $P$. emblica.

In India where $P$. emblica is well established as a commercial fruit crop, this problem is tackled through alternative vegetative propagation techniques. In addition, they predominantly depend on tissue culture techniques (Pushpakumara et al., 2007). The drawbacks in tissue culture techniques were addressed through developing in vitro shoot proliferation (Goyal \& Bhadauria, 
2007) though it is costly and cannot be implemented in breeding through sexual propagation.

The aim of this study was to identify a method to break up the seed dormancy of $P$. emblica. The proposed method would help to sever the seed dormancy and promote $P$. emblica from its underutilized status through increasing natural propagation potential and easing the breeding effort.

\section{Materials and Methods}

\section{Collection of Drupes from $P$. emblica}

The mature drupes were harvested from $P$. emblica trees in three selected areas of Anuradhapura, Kandy and Kurunegala districts, Sri Lanka from February to April 2013. From each district drupes from seven selected trees were used to obtain seeds adding up to a bulk sample of 21 trees for seed dormancy analysis.

\section{Extraction of Seeds}

The mesocarp of the drupes was cut open and completely removed (de pulped) using a knife and the stones were left to air dry for 24 hours. The stone (Figure 1) was split opened by applying a light pressure longitudinally. From each drupe up to four seeds were extracted. The extracted seeds were air dried for 24 hours.

\section{Selection of Viable Seeds}

The extracted seeds were kept in a jar containing water and were subjected to float test to determine seed viability. The seeds that sank to the bottom were selected for pre treatments.

\section{Seed Pre Treatment and Germination}

Prior to sowing, all the equipment (i.e. forceps, scalper and 3 $\mathrm{ml}$ syringe) used in sowing seeds was disinfected using $1 \%$ Clorox solution (The Clorox Company, Oakland, Canada). The four seed pre treatments used were none treated seeds (i.e. con-

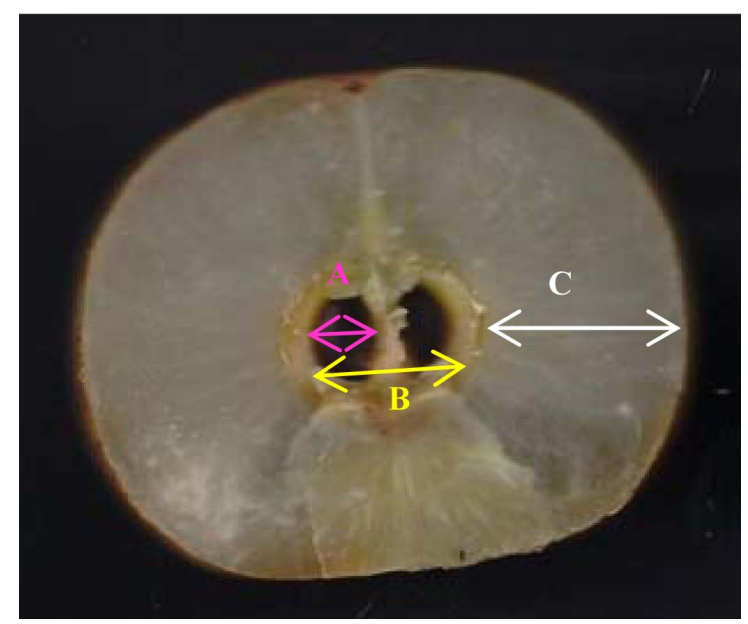

Figure 1.

Placement of seeds within the $P$. emblica stone across a longitudinal cross section, A: Seed within the stone, B: stone/hardened endocarp, C: mesocarp. The same color is used for the arrow and for the corresponding letter showing the placement of seeds within the $P$. emblica drupe. trol), seeds scarified, seeds scarified (partially removed) and treated with $1 \%$ gibberellin (Wako Pure Chemical Industries, Osaka, Japan) and seed coat removed and treated with $1 \%$ gibberelin. The seeds after pre treatments were germinated by placing on moistened paper towel. A single seed was sown in a paper towel and placed inside a zip lock bag with the mouth open and kept in a well aerated area with direct morning sunlight. The seeds were watered weekly with $1 \mathrm{ml}$ of tap water using a disinfected syringe. The seed germination was observed closely and the number of seeds that have germinated from each treatment was counted.

\section{Results}

\section{The Effect of Pre Treatments on Seed Dormancy}

The four seed pre treatments to break the seed dormancy used were untreated seeds, seeds scarified, seeds scarified (partially removed) and treated with $1 \%$ gibberellin and seed coat removed and treated with $1 \%$ gibberellin. Out of the four seed pre treatments, none of the seeds from none treated (Figure 2(A)); seeds scarified (Figure 2(B)) or seed coat removed and treated with $1 \%$ gibberellin (Figure 2(C)) showed germination even after three months. However, nine seeds that were subjected to seed scarification and $1 \%$ gibberellin pre treatment germinated exactly after 85 days of pre treatment with a germination percentage of $43 \%$ (Table 1). Complete seedlings (Figure 2(D)) with approximately $5 \mathrm{~cm}$ long radical and the cotyledon were emerged on the paper towel (inside the zip lock bag) within a week of liberating from the dormancy.

\section{Discussion}

Among the four seed pre treatments, none treated, seed coat scarified, seed coat removed and treated with $1 \%$ gibberellin did not have any effect on breaking the dormancy of $P$. emblica seeds. The highest effect on breaking the seed dormancy was shown by the seed pre treatment where the seed coat was scarified and treated with $1 \%$ gibberellin. This suggests that the natural germination potential of $P$. emblica seeds is very low. Therefore large scale $P$. emblica plantations are predominantly dependent on vegetative propagation. It can be vegetatively propagated through cutting, budding and inarching (Pushpakumara et al., 2007). But vegetative propagation is a difficult and a slow process (Goyal \& Bhadauria, 2007). Therefore many of the commercial growers of $P$. emblica are using in vitro regeneration methods (i.e. tissue culture). Through recent studies done in neighboring India, they have been able to develop triploid plantlets from the endosperm of $P$. emblica using in vitro propagation (Pushpakumara et al., 2007). Yet shoot proliferation in these in vitro explants are found to be poor (Goyal \& Bhadauria, 2007). Therefore there is an urgent need to develop

Table 1.

Germination percentages of four pre treatments.

\begin{tabular}{cc}
\hline Treatment & $\begin{array}{c}\text { Germination } \\
\text { percentage (\%) }\end{array}$ \\
\hline None treated seeds & 0 \\
Seed coat scarified & 0 \\
Seed coat scarified and treated with 1\% gibberellin & 43 \\
Seed coat removed and treated with 1\% gibberellin & 0 \\
\hline
\end{tabular}




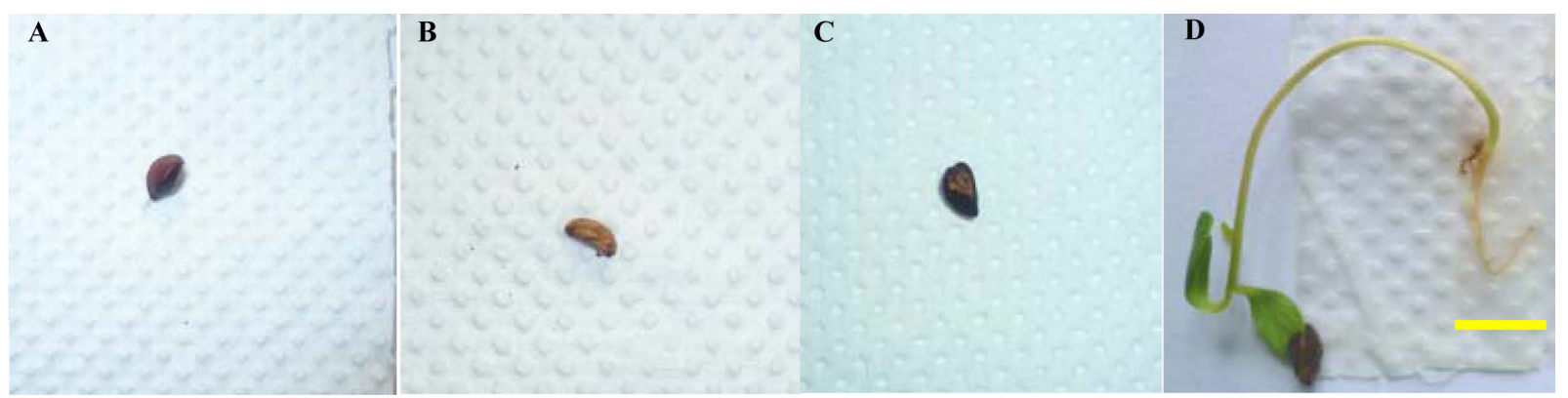

Figure 2.

Germination among pre sowing treatments of P. emblica seeds; (A) Ungerminated seed none treated; (B) Ungerminated seed pre treated with $1 \%$ gibberellin and removal of seed coat; (C) Ungerminated seed pre treated with seed scarification and (D) Seedling developed from seed pre treated with $1 \%$ gibberellin and seed scarification. Scale bar represent $1 \mathrm{~cm}$. The photos were taken after 92 days of the pre treatment and placement on the moist paper towels for germination.

a successful micro propagation protocol for $P$. emblica in order to expand its commercial market.

According to the observations made $P$. emblica seeds seem to have seed coat imposed dormancy as in peach (Mehanna \& Martin, 1985). Research conducted with Arabidopsis gibberellin deficient mutant strains indicated that, seed coat imposed dormancy can be overridden by application of gibberellin at the stage of germination (Foley, 2001). It has been found that gibbrrelin can induce the expressions and repression RGL2 (gibbrrelin-response height-regulating factors) which acts as an integrator of environmental and endogenous cues for germination (Peng \& Harberd, 2002). Therefore by manipulating the concentration of gibberellin during seed pre treatment it would be possible to change the germination percentage and reduce the time taken for germination. In species such as Penstemon digitalis the germination percentage and rate increases up to a threshold concentration of gibberellins (Mello et al., 2009). However it is inconclusive to comment that gibberellin concentration has a directly proportionate variation with germination percentage in $P$. emblica.

The seeds of $P$. emblica are enclosed within a hardened endocarp of approximately $1 \mathrm{~cm}$ in diameter (Dassanayake and Fosberg, 1986). To break the seed coat imposed physical dormancy scarification of seed coat was used. It has resulted in increasing the germination potential of many species as African Locust Bean (Aliero, 2004) and Pedicularis (Li et al., 2007). In Indian dry forests $P$. emblica seeds are dispersed by ruminants (Prasad et al., 2004). But seeds regurgitated by ruminants have a lower germination potential (22\%) than seeds which are unconsumed (72\%; Prasad et al., 2004). Other than ruminants no other species have shown frugivore in relation to $P$. emblica (Prasad et al., 2004). This could be due to the high astringency of the drupe. Therefore it is possible that the seeds of $P$. emblica undergo passive dispersal. Passive dispersal requires long term viability of the seed until the seed come in to contact with a suitable substratum. It is possible that this seed coat imposed dormancy of $P$. emblica is a safety measure to maintain the viability of seeds until landing on to an appropriate microenvironment. Thus the seeds of $P$. emblica are classified as semi orthodox (Pushpakumara et al., 2007).

In summary these observations and results shows that $P$. emblica has a high seed coat imposed dormancy which can be overridden by gibberellin pre treatment. Yet further studies are needed to assess the effect of plant growth regulators controlling the $P$. emblica seed dormancy in order to provide quality planting material for growers which will help to promote $P$. emblica from its underutilized fruit crop status.

\section{Conclusion}

The seed dormancy of $P$. emblica can be overridden by the pre treatment where the seed coat was scarified and treated with $1 \%$ gibberellin. This method can be used to germinate seeds of $P$. emblica in breeding programs and in any other studies which require seedlings.

\section{Acknowledgements}

This project was funded by University of Peradeniya, Sri Lanka Research Grant (RG/2013/15/S).

\section{REFERENCES}

Aliero, B. L. (2004). Effects of sulphuric acid, mechanical scarification and wet heat treatments on germination of seeds of African locust bean tree. Parkia biglobosa. African Journal of Biotechnology, 3, 179-181.

Dassanayake, M. D., \& Fosberg, F. R. (1988). A revised hand book of the Flora of Ceylon (pp. 219-220). New Delhi: Model Press Pvt. Ltd.

Foley, M. E. (2001). Seed dormancy: An update on terminology, physiological genetics, and quantitative trait loci regulating germinability. Weed Science, 49, 305-317. http://dx.doi.org/10.1614/0043-1745(2001)049[0305:SDAUOT]2.0. $\mathrm{CO} ; 2$

Goyal, D., \& Bhadauria, S. (2008). In vitro shoot proliferation in Emblica officinalis var. Balwant from nodal explants. Indian Journal of Biotechnology, 7, 394-397.

Krishnaveni, M., \& Mirunalini, S. (2010). Therapeutic potential of Phyllanthus emblica (amla): The ayurvedic wonder. Journal of Basic and Clinical Phyisiology and Pharmacology, 21, 93-105.

Li, A. R., Guan, K. Y., \& Probert, R. J. (2007). Effects of light, scarification, and gibberellic acid on seed germination of eight Pedicularis species from Yunnan, China. Hort Science, 42, 1259-1262.

Mehanna, H. T., \& Martin, G. C. (1985). Effect of seed coat on peach seed germination. Scientia Horticulturae, 25, 247-254. http://dx.doi.org/10.1016/0304-4238(85)90122-0

Mello, M. A., Streck, N. A., Blankenship, E. E., \& Paparozzi, E. T. (2009). Gibberellic acid promotes seed germination in Penstemon digitalis cv. Husker Red. HortSceince, 44, 870-873.

Peng, J., \& Harberd, N. P. (2002). The role of GA-mediated signalling in the control of seed germination. Current Opinion in Plant Biology, 5, 376-381. http://dx.doi.org/10.1016/S1369-5266(02)00279-0

Prasad, S., Chellam, R., Krishnaswamy, J., \& Goyal, S. P. (2004). Frugivory of Phyllanthus emblica at Rajaji Natinal Park, northwest 
India. Current Science, 87, 1188-1190.

Pushpakumara, D. K. N. G., \& Heenkenda, H. M. S. (2007). Chapter 6. Nelli (Amla) Phyllanthus emblica L. In: D. K. N. G. Pushpakumara,
H. P. M. Gunasena, \& V. P. Singh (Eds.), Underutilized fruit trees in Sri Lanka. Volume 1. World Agroforestry Centre (pp. 180-221). New Delhi: South Asia Office. 